\title{
Interventional management of central venous thromboses associated with central venous catheters: our attempts are not in vein
}

\author{
A. U. Murugananthan ${ }^{1}$, P. $\operatorname{Paskaran}^{1}$, M. M. Y. Khoo ${ }^{2}$, Y. Naji ${ }^{2}$, T. $\operatorname{Tran}^{2}$, J. M. D. Nightingale ${ }^{1}$ \\ and S. M. Gabe \\ ${ }^{1}$ Lennard-Jones Intestinal Failure Unit, St Mark's Hospital, Northwick Park, Watford Road, Harrow, Middlesex HA1 3UJ \\ and ${ }^{2}$ Radiology Department, Northwick Park Hospital, Watford Road, Harrow, Middlesex HA1 3UJ
}

Central venous $(\mathrm{CV})$ thrombosis associated with a central venous catheter (CVC) has an incidence of 0.027 episodes per catheter per year ${ }^{(1)}$ in parenteral nutrition administration. If upper limb venous access is lost, femoral access can be utilised but has a higher rate of CVC line infection ${ }^{(2)}$. Additionally loss of $\geq 2$ central veins is an indication for intestinal transplantation ${ }^{(3)}$. Guidelines exist for CVC associated $\mathrm{CV}$ thrombosis in oncology patients but they highlight the poor evidence base for attempts to treat the thrombosis and reestablish vein patency $^{(4)}$. In the parenteral nutrition literature there are only paediatric case reports ${ }^{(5)}$. We describe a 4-year single tertiary centre experience of the interventional management of CV thrombosis associated with CVCs.

Patients who underwent interventional management of their CV thromboses were identified using a radiological database. Clinical records were obtained. Catheter tip position was recorded as described by Cadman et al. ${ }^{(6)}$ Treatment outcomes were determined prior to discharge by venography.

Nine ( 8 females) patients underwent intervention for CVC associated CV thrombosis over 4 years. Mean age was 48.8 years (range 22-67) and the IF aetiology was functional (3), Crohn's disease (3), mesenteric infarction (2) and FAP/desmoid disease (1). Six patients were smokers and 2 were taking anti-coagulant therapy. Seven patients were on intravenous nutrition and 2 on parenteral fluids only. Mean duration of parenteral therapy was 4.4 years and the Broviac catheters were in situ for 19.2 months (mean, range $0.5-8$ years). Eight out of nine patients were found to have co-existing CVC sepsis. Four out of nine patients were noted to have a proximal/high line tip position at time of thrombosis.

$\mathrm{CV}$ thrombosis was located in the $\mathrm{R}$ and $\mathrm{L}$ brachiocephalic veins or above in 4 and 1 patients, respectively, and in the superior vena cava in 4 patients. All patients received local thrombolysis. Three out of nine had in addition a stent placed and 1/9 had venoplasty. One patient required local thrombolysis, venoplasty and stenting to regain patency. Outcomes at discharge were that CV patency was restored in 6/9 patients. All patients had their CVCs removed and repositioned after treatment of the acute event and were anti-coagulated longterm. Two patients developed complications of bleeding after thrombolysis (one at venepuncture site and the other developed occult GI bleeding) but required no therapy. Thirty day mortality was zero.

We show that local thrombolysis, venoplasty and venous stenting are safe methods of re-establishing venous patency. When used in combination, they re-establish venous patency in two-thirds of patients.

1. Howard L \& Ashley C (2003) Gastroenterology 124, 1651-1661.

2. Goetz AM et al. (1998) Infect Control Hosp Epidemiol. 19, 842-845.

3. American Gastroenterological Association (2003) Gastroenterology 124, 1111-1134.

4. Working Group (2009) Ann Oncol. 20(9), 1459-1491.

5. de Buys Roessingh et al. (2008) J Pediatr Surg. 43(6), E21-E24.

6. Cadman A et al. (2004) Clin Radiol 59, 349-355. 